\title{
HIGH DEGREES IN RANDOM RECURSIVE TREES
}

\author{
LOUIGI ADDARIO-BERRY AND LAURA ESLAVA
}

\begin{abstract}
For $n \geq 1$, let $T_{n}$ be a random recursive tree (RRT) on the vertex set $[n]=\{1, \ldots, n\}$. Let $\operatorname{deg}_{T_{n}}(v)$ be the degree of vertex $v$ in $T_{n}$, that is, the number of children of $v$ in $T_{n}$. Devroye and Lu [6] showed that the maximum degree $\Delta_{n}$ of $T_{n}$ satisfies $\Delta_{n} /\left\lfloor\log _{2} n\right\rfloor \rightarrow 1$ almost surely; Goh and Schmutz [7] showed distributional convergence of $\Delta_{n}-\left\lfloor\log _{2} n\right\rfloor$ along suitable subsequences. In this work we show how a version of Kingman's coalescent can be used to access much finer properties of the degree distribution in $T_{n}$.

For any $i \in \mathbb{Z}$, let $X_{i}^{(n)}=\left|\left\{v \in[n]: \operatorname{deg}_{T_{n}}(v)=\lfloor\log n\rfloor+i\right\}\right|$. Also, let $\mathcal{P}$ be a Poisson point process on $\mathbb{R}$ with rate function $\lambda(x)=2^{-x} \cdot \ln 2$. We show that, up to lattice effects, the vectors $\left(X_{i}^{(n)}, i \in \mathbb{Z}\right)$ converge weakly in distribution to $(\mathcal{P}[i, i+1), i \in \mathbb{Z})$. We also prove asymptotic normality of $X_{i}^{(n)}$ when $i=i(n) \rightarrow-\infty$ slowly, and obtain precise asymptotics for $\mathbf{P}\left(\Delta_{n}-\log _{2} n>i\right)$ when $i(n) \rightarrow \infty$ and $i(n) / \log n$ is not too large. Our results recover and extends the previous distributional convergence results on maximal and near-maximal degrees in random recursive trees.
\end{abstract}

\section{Statement of Results}

The process of random recursive trees $\left(T_{n}, n \geq 1\right)$ is defined as follows. $T_{1}$ has a single node with label 1 , which its root. The tree $T_{n+1}$ is obtained from $T_{n}$ by directing an edge from a new vertex $n+1$ to $v \in[n]$; the choice of $v$ is uniformly random and independent for each $n \in \mathbb{N}$. We call $T_{n}$ a random recursive tree (RRT) of size $n$.

As a consequence of the construction, vertex-labels in $T_{n}$ increase along root-to-leaf paths. Rooted labelled trees with such property are called increasing trees. It is not difficult to see that, in fact, $T_{n}$ is uniformly chosen among the set $\mathcal{I}_{n}$ of increasing trees with vertex set $[n]$.

We write $\operatorname{deg}_{T_{n}}(v)$ to denote the number of children of $v$ in $T_{n}$. The degree distribution of $T_{n}$ is encoded by the variables $Z_{i}^{(n)}=\left|\left\{v \in[n]: \operatorname{deg}_{T_{n}}(v)=i\right\}\right|$, for $i \geq 0$. In fact, the study of RRT's started with a paper by Na and Rapoport [13] in which they obtained, for any fixed $i \geq 0$, the convergence $\mathbb{E}\left(Z_{i}^{(n)}\right) / n \rightarrow 2^{-i-1}$ as $n \rightarrow \infty$; this result was extended to convergence in probability by Meir and Moon in [12]. Mahmoud and Smythe [11] derived the asymptotic joint normality of $Z_{i}^{(n)}$ for $i \in\{0,1,2\}$; and finally, Janson [8] extended the joint normality to $Z_{i}^{(n)}$ for $i \geq 0$ and gave explicit formulae for the covariance matrix.

The above results concern typical degrees; the focus in this work is large degree vertices, and in particular the maximum degree in $T_{n}$, which we denote $\Delta_{n}=\max _{v \in[n]} \operatorname{deg}_{T_{n}}(v)$. For the rest of the paper we write log to denote logarithms with base 2, and ln to denote natural $\operatorname{logarithms.~For~} n \in \mathbb{N}$ let $\varepsilon_{n}=\log n-\lfloor\log n\rfloor$.

A heuristic to find the order of $\Delta_{n}$ is that, if $\mathbb{E}\left(Z_{i}^{(n)}\right) \approx n 2^{-i-1}$ were to hold for all $i$, as it does when $i$ is fixed, then we would have $\mathbb{E}\left(Z_{\lfloor\log n\rfloor}^{(n)}\right) \approx n 2^{-\lfloor\log n\rfloor-1}=2^{-1+\varepsilon_{n}}$.

Date: July 4, 2016.

2010 Mathematics Subject Classification. 60C05, 05C80. 
This heuristic suggests that $\Delta_{n}$ is of order $\log n$. This is indeed the case: Szymanski [15] proved that $\mathbf{E}\left[\Delta_{n}\right] / \log n \rightarrow 1$ as $n \rightarrow \infty$, and Devroye and Lu [6] later established that $\Delta_{n} / \log n \rightarrow 1$ a.s.. Finally, Goh and Schmutz [7] showed that $\Delta_{n}-\lfloor\log n\rfloor$ converges in distribution along suitable subsequences, and identified the possible limiting laws.

Since we focus on maximal degrees, it is useful to let

$$
X_{i}^{(n)}=Z_{i+\lfloor\log n\rfloor}^{(n)}=\left|\left\{v \in[n]: \operatorname{deg}_{T_{n}}(v)=\lfloor\log n\rfloor+i\right\}\right|,
$$

for $n \in \mathbb{N}$ and $i \geq-\lfloor\log n\rfloor$. The following is a simplified version of one of our main results.

Theorem 1.1. Fix $\varepsilon \in[0,1]$. Let $\left(n_{l}\right)_{l \geq 1}$ be an increasing sequence of integers satisfying $\varepsilon_{n_{l}} \rightarrow \varepsilon$ as $l \rightarrow \infty$. Then, as $l \rightarrow \infty$

$$
\left(X_{i}^{\left(n_{l}\right)}, i \in \mathbb{Z}\right) \stackrel{\mathrm{d}}{\longrightarrow}\left(P_{i}^{\varepsilon}, i \in \mathbb{Z}\right)
$$

jointly for all $i \in \mathbb{Z}$ where the $P_{i}^{\varepsilon}$ are independent Poisson r.v.'s with mean $2^{-i-1+\varepsilon}$.

The random variables $X_{i}^{(n)}$ do not converge in distribution as $n \rightarrow \infty$ without taking subsequences; this is essentially a lattice effect caused by the floor $\lfloor\log n\rfloor$ in the definition of $X_{i}^{(n)}$.

Theorem 1.1 can be stated in terms of weak convergence of point processes (which is equivalent to convergence of finite dimensional distributions (FDD's); see Theorem 11.1.VII in [4]). In fact, we will also prove convergence (along subsequences) of

$$
X_{\geq i}^{(n)}=\sum_{k \geq i} X_{k}^{(n)}=\left|\left\{v \in[n]: \operatorname{deg}_{T_{n}}(v) \geq\lfloor\log n\rfloor+i\right\}\right| .
$$

This is useful as it yields information about $\Delta_{n}$ which cannot be derived from Theorem 1.1. We formulate this result as a statement about convergence of point processes, and now provide the relevant definitions. Let $\mathbb{Z}^{*}=\mathbb{Z} \cup\{\infty\}$. Endow $\mathbb{Z}^{*}$ with the metric defined by $\mathrm{d}(i, j)=\left|2^{-j}-2^{-i}\right|$ and $\mathrm{d}(i, \infty)=2^{-i}$ for $i, j \in \mathbb{Z}$. Let $\mathcal{M}_{\mathbb{Z}^{*}}^{\#}$ be the space of boundedly finite measures of $\mathbb{Z}^{*}$.

Let $\mathcal{P}$ be a Poisson point process on $\mathbb{R}$ with rate function $\lambda(x)=2^{-x} \cdot \ln 2$. For each $\varepsilon \in[0,1]$ let $\mathcal{P}^{\varepsilon}$ be the point process on $\mathbb{Z}^{*}$ given by

$$
\mathcal{P}^{\varepsilon}=\sum_{x \in \mathcal{P}} \delta_{\lfloor x+\varepsilon\rfloor}
$$

Similarly, for all $n \in \mathbb{N}$ let

$$
\mathcal{P}^{(n)}=\sum_{v \in[n]} \delta_{\operatorname{deg}_{T_{n}}(v)-\lfloor\log n\rfloor} .
$$

Then, for each $i \in \mathbb{Z}$ we have that

$$
\mathcal{P}^{\varepsilon}(\{i\}):=|\{x \in \mathcal{P}:\lfloor x+\varepsilon\rfloor=i\}|=|\{x \in \mathcal{P}: x \in[i-\varepsilon, i+1-\varepsilon)\}|
$$

has distribution $\operatorname{Poi}\left(2^{-i-1+\varepsilon}\right)$; also $\mathcal{P}^{(n)}(\{i\})=X_{i}^{(n)}$. We abuse notation by writing, e.g., $\mathcal{P}^{(n)}(i)=\mathcal{P}^{(n)}(\{i\})$.

It is clear that $\mathcal{P}^{(n)}$ and $\mathcal{P}^{\varepsilon}$ are elements of $\mathcal{M}_{\mathbb{Z}^{*}}^{\#}$. The advantage of working on the state space to $\mathbb{Z}^{*}$ is that intervals $[k, \infty]$ are compact. In particular, the convergence of FDD's of $\mathcal{P}^{\left(n_{l}\right)}$ implies the convergence in distribution of $X_{\geq i}^{\left(n_{l}\right)}=\mathcal{P}^{\left(n_{l}\right)}[i, \infty)$. 
Theorem 1.2. Fix $\varepsilon \in[0,1]$. Let $\left(n_{l}\right)_{l \geq 1}$ be an increasing sequence of integers satisfying $\varepsilon_{n_{l}} \rightarrow \varepsilon$ as $l \rightarrow \infty$. Then in $\mathcal{M}_{\mathbb{Z}^{*}}^{\#}, \mathcal{P}^{\left(n_{l}\right)}$ converges weakly to $\mathcal{P}^{\varepsilon}$ as $l \rightarrow \infty$. Equivalently, for any $i<i^{\prime} \in \mathbb{Z}$, jointly as $l \rightarrow \infty$

$$
\left(X_{i}^{\left(n_{l}\right)}, \ldots, X_{i^{\prime}-1}^{\left(n_{l}\right)}, X_{\geq i}^{\left(n_{l}\right)}\right) \stackrel{\mathrm{d}}{\longrightarrow}\left(\mathcal{P}^{\varepsilon}(i), \ldots, \mathcal{P}^{\varepsilon}\left(i^{\prime}-1\right), \mathcal{P}^{\varepsilon}\left[i^{\prime}, \infty\right)\right) .
$$

Note that Theorem 1.1 follows from Theorem 1.2. We finish this section stating two additional results. The first is an extension of the main theorem from [7], that result being essentially the case $i=O(1)$.

Theorem 1.3. For any $i=i(n)$ with $i+\log n<2 \ln n$ and $\liminf _{n \rightarrow \infty} i(n)>-\infty$,

$$
\mathbf{P}\left(\Delta_{n} \geq\lfloor\log n\rfloor+i\right)=\left(1-\exp \left\{-2^{-i+\varepsilon_{n}}\right\}\right)(1+o(1)) .
$$

When $i=O(1)$, the assertion of Theorem 1.3 is a straight-forward consequence of Theorem 1.2. For the case that $i(n) \rightarrow \infty$ we use estimates for the first and second moments of $X_{\geq i}^{(n)}$; note that $\left\{\Delta_{n}<\lfloor\log n\rfloor+i\right\}=\left\{X_{\geq i}^{(n)}=0\right\}$.

Finally, we also obtain the asymptotic normality for $X_{i}^{(n)}$ when $i$ tends to $-\infty$ slowly enough.

Theorem 1.4. If $i=i(n) \rightarrow-\infty$ and $i=o(\ln n)$, then as $n \rightarrow \infty$

$$
\frac{X_{i}^{(n)}-2^{-i-1+\varepsilon_{n}}}{\sqrt{2^{-i-1+\varepsilon_{n}}}} \stackrel{\mathrm{d}}{\rightarrow} N(0,1) .
$$

Remark 1.5. Up to lattice effects, Theorems 1.2 and 1.4 extend the range of $i=i(n)$ for which the heuristic that $Z_{i}^{(n)} \approx n 2^{-i-1}$ holds.

A key novelty of our approach is that for each $n$ we use Kingman's coalescent to generate a tree $T^{(n)}$ whose vertex degrees $\left\{\operatorname{deg}_{T^{(n)}}(v)\right\}_{v \in[n]}$ are exchangeable but otherwise have the same law as degrees in $T_{n}$. (See [2], Chapter 2 for a description of Kingman's coalescent, and [1], Section 2.2 for a description of the connection with random recursive trees which we exploit in this paper.) By this we mean that if $\sigma:[n] \rightarrow[n]$ is a uniformly random permutation then the following distributional identiy holds:

$$
\left(\operatorname{deg}_{T^{(n)}}(v), v \in[n]\right) \stackrel{\mathrm{d}}{=}\left(\operatorname{deg}_{T_{n}}(\sigma(v)), v \in[n]\right) .
$$

We describe the trees $T^{(n)}, n \in \mathbb{N}$ in Section 3 .

An essentially equivalent construction was used by Devroye [5] to study union-find trees. In [14], Pittel related the results of [5] on union-find trees to the height of RRT's. It is worth mentioning that both Kingman's coalescent and the union-find trees can be equivalently represented as binary trees or, as we will see in Section 3, as RRT's. Aside from the works [5] and [14], it seems that the use of Kingman's coalescent or of union-find trees to study RRT's is rare. However, it turns out to provide just the right perspective for studying high degree vertices.

\section{OutLine}

In this section we sketch the approach used in the paper. The proofs of the theorems relay on the computation of the moments of the FDD's of $\mathcal{P}^{(n)}$; these estimates are given in Proposition 2.1. In particular, the proofs of Theorems 1.2 and 1.4 use the method of moments (e.g., see [9] Section 6.1, and [3] Section 1.5).

Any FDD of $\mathcal{P}^{(n)}$ can be recovered from suitable marginals of the joint distribution of $\left(X_{i}^{\left(n_{l}\right)}, \ldots, X_{i^{\prime}-1}^{\left(n_{l}\right)}, X_{\geq i^{\prime}}^{\left(n_{l}\right)}\right)$ for some $i<i^{\prime} \in \mathbb{Z}$. For simplicity, we focus for the moment 
on collections of variables $X_{i}^{(n)}, \ldots, X_{i^{\prime}}^{(n)}$ for $i \leq i^{\prime}$. For $r \in \mathbb{R}$ and $a \in \mathbb{N}$ write $(r)_{a}=$ $r(r-1) \cdots(r-a+1)$, also let $(r)_{0}=1$. We will prove that for any non-negative integers $a_{i}, \ldots, a_{i^{\prime}}$, as $n \rightarrow \infty$, we have

$$
\mathbf{E}\left[\prod_{i \leq k \leq i^{\prime}}\left(X_{k}^{(n)}\right)_{a_{k}}\right]-\prod_{i \leq k \leq i^{\prime}}\left(2^{-(k+1)+\varepsilon_{n}}\right)^{a_{k}} \rightarrow 0 .
$$

This immediately yields Theorem 1.1.

By the linearity of expectation, proving (2) reduces to understanding the probabilities

$$
\mathbf{P}\left(\operatorname{deg}_{T_{n}}\left(v_{k}\right)=\lfloor\log n\rfloor+i_{k}, k \in[K]\right)
$$

for all $i_{1}, \ldots i_{K} \in \mathbb{N}$ and $v_{1}, \ldots v_{K} \in[n], K \in \mathbb{N}$; see Section 5 for more details.

In the standard model for RRT's described at the beginning, $\operatorname{deg}_{T_{n}}(v)$ is a sum of Bernoulli variables:

$$
\operatorname{deg}_{T_{n}}(v)=\sum_{v<u \leq n} \mathbf{1}_{\{u \rightarrow v\}} .
$$

The lack of symmetry of the degrees $\left\{\operatorname{deg}_{T_{n}}(v)\right\}_{v \in[n]}$ complicates the analysis of (3). In proving that $\Delta_{n} / \log n \stackrel{\text { a.s. }}{\rightarrow} 1$, Devroye and Lu [6] used that $\left\{\operatorname{deg}_{T_{n}}(v)\right\}_{v \in[n]}$ are negatively orthant dependent (see [10] for a definition), which in particular means that for all $S \subset[n]$ and $m_{1}, \ldots, m_{n} \in \mathbb{N}$

$$
\mathbf{P}\left(\operatorname{deg}_{T_{n}}(v) \geq m_{v}, v \in S\right) \leq \prod_{v \in S} \mathbf{P}\left(\operatorname{deg}_{T_{n}}(v) \geq m_{v}\right)
$$

and then obtained upper bounds for $\mathbf{P}\left(\operatorname{deg}_{T_{n}}(v) \geq c \ln n\right)$ for each $v \in[n]$.

One approach to studying high degrees in $T_{n}$ would be to obtain matching lower bounds for $\mathbf{P}\left(\operatorname{deg}_{T_{n}}(v) \geq m_{v}, v \in S\right)$, with uniform error terms even when $m_{v}$ is large. Instead, we study trees $T^{(n)}$, mentioned in (1), above, for which we can obtain precise asymptotics for the analogous probabilities

$$
\mathbf{P}\left(\operatorname{deg}_{T^{(n)}}(v) \geq m_{v}, v \in[K]\right) .
$$

The core of the paper lies in Proposition 4.2, which gives precise estimates of (5) for $m_{1}, \ldots, m_{K}$ in a suitable range. Broadly speaking, $\operatorname{deg}_{T^{(n)}}(v)$ depends on a set of random selection times $\mathcal{S}_{v}$ and the first streak of heads in a sequence of $\left|\mathcal{S}_{v}\right|$ fair coin flips. As mentioned in the previous section, the degrees of $T^{(n)}$ have the same distribution as the degrees in $T_{n}$. Consequently, our estimation of (5) allows us to obtain the following moments estimate.

Proposition 2.1. For all $c \in(0,2)$ and $K \in \mathbb{N}$ there is $\alpha=\alpha(c, K)>0$ such that the following holds. Fix any integers $i, i^{\prime}$ with $0<i+\log _{n}<i^{\prime}+\log _{n}<c \ln n$. Then for any non-negative integers $a_{i}, \ldots, a_{i^{\prime}}$ with $a_{i}+\ldots+a_{i^{\prime}}=K$, we have

$$
\mathbf{E}\left[\left(X_{\geq i^{\prime}}^{(n)} a_{i^{\prime}} \prod_{i \leq k<i^{\prime}}\left(X_{k}^{(n)}\right)_{a_{k}}\right]=\left(2^{-i^{\prime}+\varepsilon_{n}}\right)^{a_{i^{\prime}}} \prod_{i \leq k<i^{\prime}}\left(2^{-(k+1)+\varepsilon_{n}}\right)^{a_{k}}\left(1+o\left(n^{-\alpha}\right)\right) .\right.
$$

Equipped with Proposition 2.1, the proofs of the theorems are straightforward. The rest of the paper is organized as follows. In Section 3, we explain how to define the trees $T^{(n)}$ using Kingman's coalescent and establish the distributional relation between $T^{(n)}$ and the RRT; see Corollary 3.4. In Section 4, we define the random sets $\left(\mathcal{S}_{v}, v \in T^{(n)}\right)$ and explain their relation with degrees in $T^{(n)}$. The proof of Proposition 4.2, which is our estimate of 
(5), is then presented using a decoupling of the events in (5) and the concentration of the random variables $\left|\mathcal{S}_{v}\right|$. Finally, the proof of Proposition 2.1 is given in Section 5 and the proof of Theorems 1.2-1.4 are in Section 6.

\section{Random Recursive Trees and Kingman's coalescent}

In this section we give a representation of Kingman's coalescent in terms of labelled forests, and relate it to RRT's. All trees in the remainder of the paper are rooted, and we write $r(t)$ for the root of tree $t$. By convention, edges of a tree are directed towards the root of the tree and we write $u v$ to denote an edge directed from $u$ to $v$. A forest $f$ is a set of trees whose vertex sets are pairwise disjoint. The vertex set of a forest, denoted $V(f)$, is the union of the vertex sets of its trees. Similarly, $E(f)$ denotes the set of edges in the trees of $f$. For $n \geq 1$, let

$$
\mathcal{F}_{n}=\{f: V(f)=[n]\}
$$

be the set of forests with vertex set $[n]$.

A sequence $C=\left(f_{1}, \ldots, f_{n}\right)$ of elements of $\mathcal{F}_{n}$ is an $n$-chain if $f_{1}$ is the forest in $\mathcal{F}_{n}$ with $n$ one-vertex trees and, for $1 \leq i<n, f_{i+1}$ is obtained from $f_{i}$ by adding a directed edge between the roots of some pair of trees in $f_{i}$. If $\left(f_{1}, \ldots, f_{n}\right)$ is an $n$-chain then for $1 \leq i \leq n$, the forest $f_{i}$ consists of $n+1-i$ trees, and in this case we list its elements in increasing order of their smallest-labelled vertex as $t_{1}^{(i)}, \ldots, t_{n+1-i}^{(i)}$.

Definition 3.1. Kingman's $n$-coalescent is the random $n$-chain $\mathbf{C}=\left(F_{1}, \ldots, F_{n}\right)$ built as follows. Independently for each $1 \leq i \leq n-1$ let $\left\{a_{i}, b_{i}\right\}$ be a random pair uniformly chosen from $\{\{a, b\}: 1 \leq a<b \leq n+1-i\}$ and let $\xi_{i}$ be independent with Bernoulli(1/2) distribution.

For $1 \leq i<n$, construct $F_{i+1}$ from $F_{i}$ as follows. If $\xi_{i}=1$ then add an edge from $r\left(T_{b_{i}}^{(i)}\right)$ to $r\left(T_{a_{i}}^{(i)}\right)$ and if $\xi_{i}=0$ then add an edge from $r\left(T_{a_{i}}^{(i)}\right)$ to $r\left(T_{b_{i}}^{(i)}\right)$. The forest $F_{i+1}$ consists of the new tree and the remaining $n-1-i$ unaltered trees from $F_{i}$.

For an example of the process see Figure 1.

Lemma 3.2. Let $\mathcal{C F}_{n}$ be the set of $n$-chains of elements in $\mathcal{F}_{n}$. Then $\left|\mathcal{C F}_{n}\right|=n !(n-1)$ ! and Kingman's n-coalescent is a uniformly random element of $\mathcal{C F}_{n}$.

Proof. Fix an $n$-chain $\left(f_{1}, \ldots, f_{n}\right) \in \mathcal{C F}_{n}$. Then

$$
\mathbf{P}\left(\left(F_{1}, \ldots, F_{n}\right)=\left(f_{1}, \ldots, f_{n}\right)\right)=\prod_{k=1}^{n-1} \mathbf{P}\left(F_{k+1}=f_{k+1} \mid F_{j}=f_{j}, 1 \leq j \leq k\right) .
$$

Among the $(n+1-k)(n-k)$ possible oriented edges between roots of $f_{k}$, there is exactly one whose addition yields $f_{k+1}$. It follows that the $k$-th term in the above product is $((n+1-k)(n-k))^{-1}$, so $\mathbf{P}\left(\left(F_{1}, \ldots, F_{n}\right)=\left(f_{1}, \ldots, f_{n}\right)\right)=[n !(n-1) !]^{-1}$. The result follows since this expression does not depend on $\left(f_{1}, \ldots, f_{n}\right) \in \mathcal{C} \mathcal{F}_{n}$.

Recall that $\mathcal{I}_{n}$ is the set of increasing trees with vertex set $[n]$. It is not difficult to see that $\left|\mathcal{I}_{n}\right|=(n-1)$ ! and that a RRT is a uniformly random element of $\mathcal{I}_{n}$.

There is a natural mapping $\phi$ between $n$-chains and increasing trees. Given an $n$-chain $C=\left(f_{1}, \ldots, f_{n}\right)$, write $t^{(n)}:=t_{1}^{(n)}$ for the unique tree in $f_{n}$. Let $L_{C}^{-}: E\left(t^{(n)}\right) \rightarrow[n-1]$ be defined as follows. For each $e \in E\left(t^{(n)}\right)$, let

$$
L_{C}^{-}(e)=\max \left\{i \in[n-1]: e \notin E\left(t^{(i)}\right)\right\} .
$$



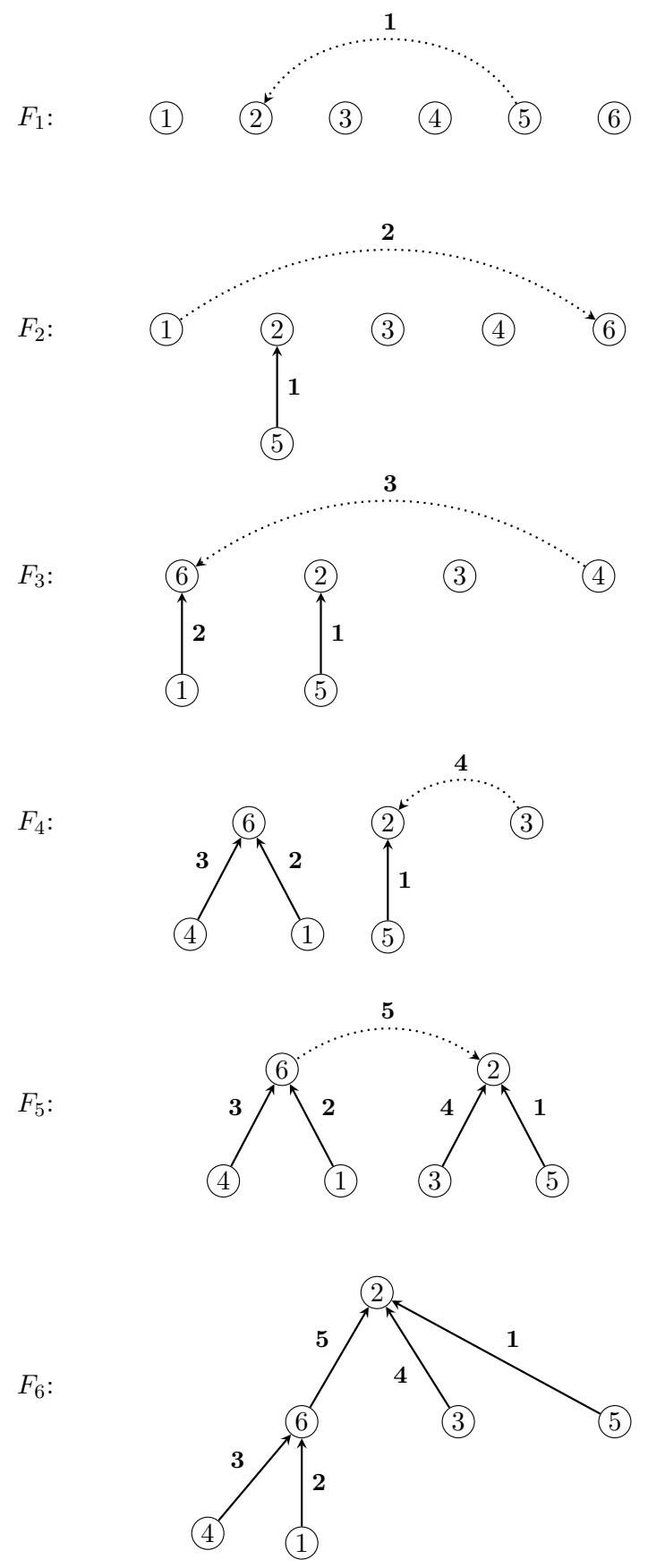

Figure 1. An example of Kingman's $n$-coalescent $\mathbf{C}=\left(F_{1}, \ldots, F_{n}\right)$ for $n=6$. For $1 \leq i<n, F_{i}$ has, in dotted line, the edge in $E\left(F_{i+1}\right) \backslash$ $E\left(F_{i}\right)$. Edges are marked with their time of addition; this is the function $L_{\mathbf{C}}^{-}$defined after Lemma 3.2. In this instance, $\xi_{1}=\xi_{3}=\xi_{4}=1, \xi_{2}=\xi_{5}=0$ and $\left\{a_{1}, b_{1}\right\}=\{2,5\}, \quad\left\{a_{2}, b_{2}\right\}=\{1,5\}, \quad\left\{a_{3}, b_{3}\right\}=\{1,4\},\left\{a_{4}, b_{4}\right\}=$ $\{2,3\},\left\{a_{5}, b_{5}\right\}=\{1,2\}$. 


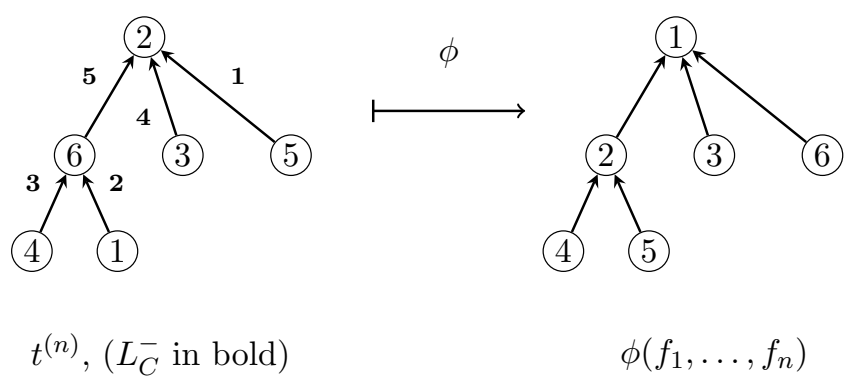

Figure 2. On the left a tree $t^{(n)}$; edges are marked with $L_{C}^{-}$, from which the $n$-chain $C=\left(f_{1}, \ldots, f_{n}\right)$ can be recovered. On the right, the increasing tree $\phi\left(f_{1}, \ldots, f_{n}\right)$; it has the shape of $t^{(n)}$ and the vertex labels $\left\{L_{C}(v), v \in\right.$ $\left.V\left(t^{(n)}\right)\right\}$.

We think of $L_{C}^{-}$as a function that keeps track of the time of addition of the edges along the $n$-chain $C$. Now, we define a vertex labelling $L_{C}: V\left(t^{(n)}\right) \rightarrow[n]$ as follows. Let $L_{C}\left(r\left(t^{(n)}\right)\right)=1$ and for each $u v \in E\left(t^{(n)}\right)$, let

$$
L_{C}(u)=n+1-L_{C}^{-}(u v)
$$

then $L_{C}(u)$ is the number of trees in the forest just before $u v$ is added.

Note that for each $i \in[n-1]$, the new edge in $f_{i+1}$ joins the roots of two trees in $f_{i}$ and is directed towards the root of the resulting tree. Thus, the labels $\left\{L_{C}^{-}(e), e \in\right.$ $\left.E\left(t^{(n)}\right)\right\}$ increase along all paths in $t^{(n)}$ towards the root $r\left(t^{(n)}\right)$ and consequently, the labels $\left\{L_{C}(v), v \in V\left(t^{(n)}\right)\right\}$ increase along root-to-leaf paths in $t^{(n)}$. This shows that relabelling the vertices of $t^{(n)}$ with $L_{C}$ yields an increasing tree (specifically, an element of $\mathcal{I}_{n}$ ). See Figure 2 for an example.

Proposition 3.3. Let $\phi: \mathcal{C F} \rightarrow \mathcal{I}_{n}$ be defined as follows. For an $n$-chain $C=\left(f_{1}, \ldots, f_{n}\right)$ let $\phi(C)$ be the tree obtained from $t^{(n)}$ by relabelling its vertices with $L_{C}$. Then $\phi(\mathbf{C})$, the push-forward of Kingman's n-coalescent by $\phi$, has the law of a RRT of size $n$.

Proof. First, we prove that $\phi$ is onto. Fix an increasing tree $t \in \mathcal{I}_{n}$. For each $j \in V(t) \backslash\{1\}$, let $v_{j} \in V(t)$ be such that $j v_{j} \in E(t)$, recall that edges are directed toward the root of $t$, thus $v_{j}$ is uniquely defined. For each $1<j \leq n$, let $e_{n-j+1}=j u_{j}$.

Now construct an $n$-chain $C$ as follows. Let $f_{1}$ be the forest with $n$ one-vertex trees. For each $1<i \leq n$ construct $f_{i}$ from $f_{i-1}$ by adding the edge $e_{i-1}$. In other words, for each $1 \leq i<n, L_{C}^{-}\left(e_{i}\right)=i$ and so $L_{C}(n+1-i)=n+1-L_{C}^{-}\left(e_{i}\right)=n+1-i$; also since $r(t)=1$, we have $L_{C}(1)=1$. Consequently, $\phi(C)=t$.

We claim that $\left|\phi^{-1}(t)\right| \geq n$ ! for any $t \in \mathcal{I}_{n}$. To see this, consider an $n$-chain $C$ and a permutation $\sigma:[n] \rightarrow[n]$. Let $C_{\sigma}$ be the $n$-chain obtained from $C$ by permuting the vertices in each forest of $C$ by $\sigma$. Since $L_{C}(v)$ depends only on the time of addition of its outgoing edge (if any), it follows that $\phi(C)=\phi\left(C_{\sigma}\right)$ for all permutations $\sigma$. By Lemma 3.2, this shows that $\phi$ is $n$ !-to- 1 and that $\phi(\mathbf{C})$ is a uniform element in $\mathcal{I}_{n}$.

Since $\phi(\mathbf{C})$ preserves the shape of $T^{(n)}$ and only relabels its vertices, the degrees in $T^{(n)}$ and $\phi(\mathbf{C})$ are equal as multisets: $\left\{\operatorname{deg}_{T^{(n)}}(v)\right\}_{v \in[n]}=\left\{\operatorname{deg}_{\phi(\mathbf{C})}(v)\right\}_{v \in[n]}$. This immediately gives the following key corollary of Proposition 3.3, on which the rest of the paper relies. 

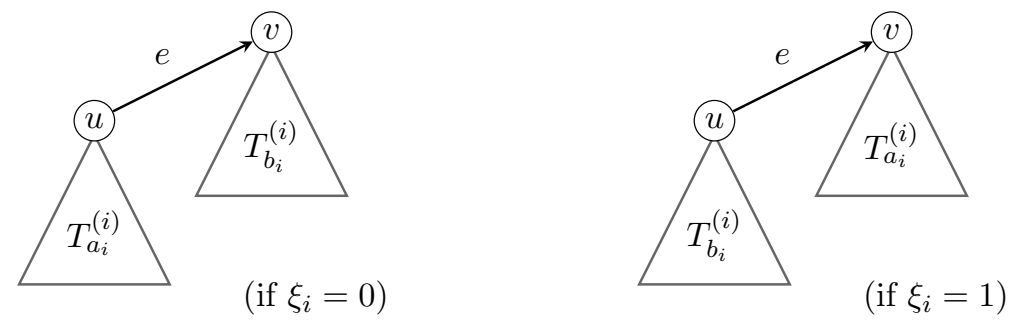

Figure 3. If $v$ is a root in $T_{a_{i}}^{(i)} \cup T_{b_{i}}^{(i)}$ and $\xi_{i}$ favours $v$, then $v$ increases its degree and remains a root in $F_{i+1}$.

Corollary 3.4. For all $n \in \mathbb{N}$, we have the following equality in distribution holds jointly for all $i \in \mathbb{Z}$,

$$
X_{i}^{(n)} \stackrel{\mathrm{d}}{=}\left|\left\{v \in[n]: \operatorname{deg}_{T^{(n)}}(v)=\lfloor\log n\rfloor+i\right\}\right| .
$$

We now proceed to the study of the joint distribution of the vertex degrees in $T^{(n)}$.

\section{Degree Distribution: Selection Sets And coin flips}

By construction, the vertex degrees $\left\{\operatorname{deg}_{T^{(n)}}(v)\right\}_{v \in[n]}$ are exchangeable. Our next goal is to explain how to approximate (5); that is, for any fixed $k \in \mathbb{N}$ and integers $m_{1}, \ldots, m_{k}<$ $2 \ln n$, to obtain estimates for $\mathbf{P}\left(\operatorname{deg}_{T^{(n)}}(v) \geq m_{v}, v \in[k]\right)$.

The key to analyse the degrees in $T^{(n)}$ is to understand how the degrees of a vertex $v \in[n]$ change in Kingman's coalescent $\mathbf{C}=\left(F_{1}, \ldots, F_{n}\right)$. For any vertex $v$ and $1 \leq i \leq n$, denote $\operatorname{deg}_{F_{i}}(v)$ the number of children of $v$ in $F_{i}$. Also, we will simply write $\operatorname{deg}(v)=\operatorname{deg}_{F_{n}}(v)=$ $\operatorname{deg}_{T^{(n)}}(v)$. For each $1 \leq i<n$, if $\xi_{i}=1$ we say that $\xi_{i}$ favours the vertices of $T_{a_{i}}^{(i)}$, and otherwise that it favours the vertices of $T_{b_{i}}^{(i)}$. For $v \in[n]$, let

$$
\mathcal{S}_{v}=\left\{i \in[n-1]: v \in T_{a_{i}}^{(i)} \cup T_{b_{i}}^{(i)}\right\} .
$$

For any vertex $v$, and $1 \leq i<n, \operatorname{deg}_{F_{i+1}}(v)$ increases by one only if $v$ is a root in $F_{i}$, $i \in \mathcal{S}_{v}$ and $\xi_{i}$ favours $v$; see Figure 3. Conversely, let $p_{v}=\min \left\{i \in \mathcal{S}_{v}, \xi_{i}\right.$ does not favour $\left.v\right\}$, then the first $F_{i+1}$ in which $v$ is not a root is exactly $i=p_{v}$. In this case, in $F_{p_{v}+1}$ there is an outgoing edge from $v$, and $v$ is not a root of any subsequent forests. As a consequence, $\operatorname{deg}_{F_{j}}(v)=\operatorname{deg}_{F_{p_{v}}}(v)$ for $p_{v}<j \leq n$.

Fact 4.1. For $v \in[n], \operatorname{deg}(v)=\operatorname{deg}_{F_{p_{v}}}(v)=\left|\mathcal{S}_{v} \cap\left[p_{v}-1\right]\right|$.

In other words, $\operatorname{deg}(v)$ depends only on its first streak of favourable random variables $\xi_{i}$ with $i \in \mathcal{S}_{v}$. More precisely, given $\left|\mathcal{S}_{v}\right|$, the degree $\operatorname{deg}(v)$ is distributed as $\min \left\{\left|\mathcal{S}_{v}\right|, G\right\}$, where $G$ is a Geometric(1/2) r.v. independent of $\mathcal{S}_{v}$.

Thus, it is relevant to observe that $\left|\mathcal{S}_{v}\right|$ is distributed as an sum of independent (though not identically distributed) Bernoulli random variables and so it is concentrated around its mean $\mathbf{E}\left[\left|\mathcal{S}_{v}\right|\right]=2 \ln n+O(1)$; a more precise statement can be found in Proposition 4.5 below. Since $\left|\mathcal{S}_{v}\right| \rightarrow \infty$ in probability as $n \rightarrow \infty$, it follows easily that $\operatorname{deg}(v)$ is asymptotically geometric for any fixed node $v$. More strongly, the following proposition shows that for any fixed $k$, the random variables $\left\{\operatorname{deg}_{T^{(n)}}(v)\right\}_{v \in[k]}$ asymptotically behave like independent Geometric random variables, even if they are conditioned to be quite large. 
Proposition 4.2. Fix $c \in(0,2)$ and $k \in \mathbb{N}$. There exists $\alpha=\alpha(c, k)>0$ such that uniformly over positive integers $m_{1}, \ldots, m_{k}<c \ln n$,

$$
\mathbf{P}\left(\operatorname{deg}_{T^{(n)}}(v) \geq m_{v}, v \in[k]\right)=2^{-\sum_{v} m_{v}}\left(1+o\left(n^{-\alpha}\right)\right) .
$$

We now explain how the events in the proposition above can be decoupled into a product of two probabilities, one of them corresponding to tail bounds for the random variables $\left|\mathcal{S}_{v}\right|$. We start with an upper bound for Proposition 4.2.

Lemma 4.3. For any $k \in \mathbb{N}$ and positive integers $m_{1}, \ldots, m_{k}<n$,

$$
\mathbf{P}\left(\operatorname{deg}(v) \geq m_{v}, v \in[k]\right) \leq 2^{-\sum_{v} m_{v}} \mathbf{P}\left(\left|\mathcal{S}_{v}\right| \geq m_{v}, v \in[k]\right) .
$$

Equality holds for $k=1$.

Proof. For each $v \in[k]$ list $\mathcal{S}_{v}$ in increasing order as $\left(i_{v, j}, 1 \leq j \leq\left|\mathcal{S}_{v}\right|\right)$. Let $\mathcal{A}$ be the set of sequences $A=\left(A_{1}, \ldots, A_{k}\right)$ satisfying $A_{v} \subset[n-1]$ and $\left|A_{v}\right|=m_{v}$ for all $v \in[k]$. For every $A \in \mathcal{A}$, let $D_{A}$ be the event that $\left|\mathcal{S}_{v}\right| \geq m_{v}$ and $\left\{i_{v, 1}, \ldots, i_{v, m_{v}}\right\}=A_{v}$, for all $v \in[k]$. By Fact 4.1 , if $\operatorname{deg}(v) \geq m_{v}$ then necessarily $\left|\mathcal{S}_{v}\right| \geq m_{v}$ so

$$
\left\{\operatorname{deg}(v) \geq m_{v}, v \in[k]\right\} \cap D_{A}=\left\{\xi_{i_{v, j}} \text { favours } v \text { for all } j \in\left[m_{v}\right], v \in[k]\right\} \cap D_{A} .
$$

Now, $\xi_{i}$ are i.i.d Bernoulli(1/2) r.v.'s. Thus, if $D_{A}$ has positive probability then

$\mathbf{P}\left(\xi_{i_{v, j}}\right.$ favours $v$ for all $\left.j \in\left[m_{v}\right], v \in[k] \mid D_{A}\right)= \begin{cases}2^{-\sum_{v} m_{v}} & \text { if }\left|A_{u} \cap A_{v}\right|=0, \forall u \neq v \in[k] \\ 0 & \text { o.w. }\end{cases}$

The second case follows from the fact that if $i \in \mathcal{S}_{u} \cap \mathcal{S}_{v}$ for some $u \neq v$, then $\xi_{i}$ cannot favour both $u$ and $v$. The events $\left(D_{A}, A \in \mathcal{A}\right)$ are pairwise disjoint, and if $\operatorname{deg}(v) \geq m_{v}$ for all $v \in[k]$ then one of the events $D_{A}$ must occur. It follows that

$$
\begin{aligned}
\mathbf{P}\left(\operatorname{deg}(v) \geq m_{v}, v \in[k]\right) & =\sum_{A \in \mathcal{A}} \mathbf{P}\left(D_{A}, \operatorname{deg}(v) \geq m_{v}, v \in[k]\right) \\
& \leq \sum_{A \in \mathcal{A}} 2^{-\sum_{v} m_{v}} \mathbf{P}\left(D_{A}\right) \\
& =2^{-\sum_{v} m_{v}} \mathbf{P}\left(\left|S_{v}\right| \geq m_{v}, v \in[k]\right)
\end{aligned}
$$

Finally, the second line holds with equality when $k=1$.

For the lower bound we restrict to events $D_{A}$ where the sets $A_{v}$ are already disjoint. To do so, we consider instead the vertex degrees in $F_{I}$ for some $I<n$. For $k \geq 2$ let

$$
\tau_{k}=\min \left\{i \in[n-1]:\left\{a_{i}, b_{i}\right\} \subset[k]\right\} .
$$

Since $F_{i} \subset F_{j}$ for all $i \leq j \in[n]$ we have that for any $I<n$

$$
\begin{aligned}
\mathbf{P}\left(\operatorname{deg}(v) \geq m_{v}, v \in[k]\right) & \geq \mathbf{P}\left(\operatorname{deg}_{F_{I+1}}(v) \geq m_{v}, v \in[k]\right) \\
& \geq \mathbf{P}\left(I<\tau_{k}, \operatorname{deg}_{F_{I+1}}(v) \geq m_{v}, v \in[k]\right) .
\end{aligned}
$$

Recall that trees in $F_{i}$ are listed in increasing order of their least elements; this implies that indices of the trees of vertices $1, \ldots, k$ do not change until two trees indexed by $a, b \leq$ $k$ are merged. Therefore, for all $v \in[k], v \in T_{v}^{(i)}$ for $i \leq \tau_{k}$. This implies the sets $\left\{\mathcal{S}_{v} \cap\left[\tau_{k}-1\right], v \in[k]\right\}$ are pairwise disjoint. These observations allow us to obtain a lower bound analogous to Lemma 4.3. 
Lemma 4.4. For any positive integers $k \geq 2$ and $m_{1}, \ldots, m_{k}, I<n$,

$$
\mathbf{P}\left(\operatorname{deg}(v) \geq m_{v}, v \in[k]\right) \geq 2^{-\sum_{v} m_{v}} \mathbf{P}\left(I<\tau_{k},\left|S_{v} \cap[I]\right| \geq m_{v}, v \in[k]\right) .
$$

Proof. By (6), it suffices to bound $\mathbf{P}\left(I<\tau_{k}, \operatorname{deg}_{F_{I+1}}(v) \geq m_{v}, v \in[k]\right)$.

Let $\mathcal{A}^{*}$ be the set of sequences $A=\left(A_{1}, \ldots, A_{k}\right)$ of pairwise disjoint subsets of $[I]$ satisfying $\left|A_{v}\right|=m_{v}$ for all $v \in[k]$. For each $A \in \mathcal{A}^{*}$, let $D_{A}$ be the event that for all $v \in[k],\left\{i_{v, j}, \ldots, i_{v, m_{v}}\right\}=A_{v}$ (and so $\left|\mathcal{S}_{v} \cap[I]\right| \geq m_{v}$ ).

As in the proof of Lemma 4.3, we have that

$$
\left\{\operatorname{deg}_{F_{I+1}}(v) \geq m_{v}, v \in[k]\right\} \cap D_{A}=\left\{\xi_{i_{v, j}} \text { favours } v \text { for all } j \in\left[m_{v}\right], v \in[k]\right\} \cap D_{A} .
$$

In this case, the sets $A_{v}$ are pairwise disjoint. If $\mathbf{P}\left(D_{A}\right)>0$ then

$$
\mathbf{P}\left(\xi_{i_{v, j}} \text { favours } v \text { for all } j \in\left[m_{v}\right], v \in[k] \mid D_{A}\right)=2^{-\sum_{v} m_{v}} .
$$

Recall that $I<\tau_{k}$ if and only if the sets $\left\{S_{v} \cap[I], v \in[k]\right\}$ are pairwise disjoint; that is, if one of the events $D_{A}$ occur. We then have

$$
\begin{aligned}
\mathbf{P}\left(I<\tau_{k}, \operatorname{deg}_{F_{I+1}}(v) \geq m_{v}, v \in[k]\right) & =\sum_{A \in \mathcal{A}^{*}} \mathbf{P}\left(D_{A}, \operatorname{deg}_{F_{I+1}}(v) \geq m_{v}, v \in[k]\right) \\
& =\sum_{A \in \mathcal{A}^{*}} 2^{-\sum_{v} m_{v}} \mathbf{P}\left(D_{A}\right) \\
& =2^{-\sum_{v} m_{v}} \mathbf{P}\left(I<\tau_{k},\left|S_{v} \cap[I]\right| \geq m_{v}, v \in[k]\right) .
\end{aligned}
$$

To use Lemma 4.4 we need tail bounds for $\left|\mathcal{S}_{v} \cap[I]\right|$ for some suitable $I<n$; these are provided by the following proposition.

Proposition 4.5. Fix $\varepsilon \in(0,1)$ and $c \in(0,2(1-\varepsilon))$. Then there exists $\beta=\beta(c, \varepsilon)>0$ such that for any vertex $v$,

$$
\mathbf{P}\left(\left|\mathcal{S}_{v} \cap\left[n-\left\lceil n^{\varepsilon}\right\rceil\right]\right|<c \ln n\right)=o\left(n^{-\beta}\right) .
$$

Proof. Fix $\varepsilon \in(0,1)$ and $c \in(0,2(1-\varepsilon))$. Let $\left\{B_{i}, i \in \mathbb{N}\right\}$ be a collection of independent Bernoulli r.v.'s, with $\mathbf{E}\left[B_{i}\right]=\frac{2}{i}$. Recall the definition of $\mathcal{S}_{v}$ at the beginning of the section.

For any fixed vertex $v \in[n]$, and each $i \in[n-1]$, the probability of the event $\{v \in$ $\left.T_{a_{i}}^{(i)} \cup T_{b_{i}}^{(i)}\right\}$ is $2 /(n-i+1)$; this is because, in the forest $F_{i}$, there are $n-i+1$ trees and the trees $T_{a_{i}}^{(i)}, T_{b_{i}}^{(i)}$ are chosen uniformly at random among them. Since each of these events are independent we have $\left|\mathcal{S}_{v}\right| \stackrel{\mathrm{d}}{=} \sum_{i=2}^{n} B_{i}$. Moreover, writing $W_{n, \varepsilon}=\sum_{i=n-\left\lceil n^{\varepsilon}\right\rceil}^{n} B_{i}$, we also have

$$
W_{n, \varepsilon} \stackrel{\mathrm{d}}{=}\left|\mathcal{S}_{v} \cap\left[n-\left\lceil n^{\varepsilon}\right\rceil\right]\right| .
$$

We now apply Bernstein's inequality (see, e.g., [9], Theorem 2.8) to obtain that for any $t>0$

We take $t=\mathbf{E}\left[W_{n, \varepsilon}\right]-c \ln n$. Since

$$
\mathbf{P}\left(W_{n, \varepsilon} \leq \mathbf{E}\left[W_{n, \varepsilon}\right]-t\right) \leq \exp \left\{-\frac{t^{2}}{2 \mathbf{E}\left[W_{n, \varepsilon}\right]}\right\} .
$$

$$
\mathbf{E}\left[W_{n, \varepsilon}\right]=\sum_{i=n-\left\lceil n^{\varepsilon}\right\rceil}^{n} \frac{2}{i}=2(1-\varepsilon) \ln n+O(1),
$$

setting $\delta=2(1-\varepsilon)-c>0$ we have $t=\delta \ln n+O(1)$, so

$$
\mathbf{P}\left(\left|\mathcal{S}_{v} \cap\left[n-\left\lceil n^{\varepsilon}\right\rceil\right]\right|<c \ln n\right)=\mathbf{P}\left(W_{n, \varepsilon} \leq \mathbf{E}\left[W_{n, \varepsilon}\right]-t\right)=O(1) \cdot n^{-\delta^{2} /(4(1-\varepsilon))} .
$$


Choosing $0<\beta<\delta^{2} / 4(1-\varepsilon)$, the result follows.

The following lemma is the last ingredient for Proposition 4.2.

Lemma 4.6. Fix an integer $k \geq 2$ and let $\varepsilon \in(0,1)$. Then, for $n$ large enough,

$$
\mathbf{P}\left(\tau_{k} \leq n-\left\lceil n^{\varepsilon}\right\rceil\right) \leq \frac{2 k^{2}}{\left\lceil n^{\varepsilon}\right\rceil-1} .
$$

Proof. By the definition of $\tau_{k}$, if $\tau_{k}>n-\left\lceil n^{\varepsilon}\right\rceil$ then $\left\{a_{i}, b_{i}\right\} \not \subset[k]$ for all $1 \leq i \leq n-\left\lceil n^{\varepsilon}\right\rceil$. The events that $\left\{a_{i}, b_{i}\right\} \not \subset[k]$ are independent for distinct $i$ and $\mathbf{P}\left(\left\{a_{i}, b_{i}\right\} \subset[k]\right)=\frac{k(k-1)}{(n+1-i)(n-i)}$, so we have that

$$
\mathbf{P}\left(\tau_{k}>n-\left\lceil n^{\varepsilon}\right\rceil\right)=\prod_{i=1}^{n-\left\lceil n^{\varepsilon}\right\rceil}\left(1-\frac{k(k-1)}{(n+1-i)(n-i)}\right) \geq 1-\sum_{i=1}^{n-\left\lceil n^{\varepsilon}\right\rceil} \frac{2 k^{2}}{(n-i)^{2}}
$$

The last inequality holds for $n$ large enough. Since $\sum_{j=m}^{\infty} j^{-2} \leq \int_{m-1}^{\infty} x^{-2} d x=(m-1)^{-1}$, we get

$$
\mathbf{P}\left(\tau_{k} \leq n-\left\lceil n^{\varepsilon}\right\rceil\right) \leq \sum_{i=1}^{n-\left\lceil n^{\varepsilon}\right\rceil} \frac{2 k^{2}}{(n-i)^{2}} \leq \sum_{j=\left\lceil n^{\varepsilon}\right\rceil}^{\infty} \frac{2 k^{2}}{j^{2}}=\frac{2 k^{2}}{\left\lceil n^{\varepsilon}\right\rceil-1} .
$$

We finish this section with the proof of Proposition 4.2.

Proof of Proposition 4.2. Fix $c \in(0,2), k \in \mathbb{N}$ and let $m_{1}, \ldots, m_{k}<c \ln n$ be positive integers. Let $\varepsilon=(2-c) / 4$ so that Proposition 4.5 holds for some $\beta(c)=\beta(c, \varepsilon)>0$. For $k=1$, the result follows from the equality in Lemma 4.3 and Proposition 4.5 since

$$
\mathbf{P}\left(\left|\mathcal{S}_{1}\right|<m_{1}\right) \leq \mathbf{P}\left(\left|\mathcal{S}_{1} \cap\left[n-\left\lceil n^{\varepsilon}\right\rceil\right]\right|<c \ln n\right)=o\left(n^{-\beta}\right) .
$$

For $k \geq 2$, the upper bound is likewise established immediately by Lemma 4.3. For the lower bound, letting $I=n-\left\lceil n^{\varepsilon}\right\rceil$, by Lemma 4.6 and Proposition 4.5 we have

$\mathbf{P}\left(I<\tau_{k},\left|\mathcal{S}_{v} \cap[I]\right| \geq m_{v}, v \in[k]\right) \geq 1-\mathbf{P}\left(I \geq \tau_{k}\right)-\sum_{v \in[k]} \mathbf{P}\left(\left|\mathcal{S}_{v} \cap[I]\right|<m_{v}\right) \geq 1-o\left(n^{-\alpha}\right)$,

where $\alpha<\min \{\beta, \varepsilon\}$. By Lemma 4.4, it follows that

$$
\mathbf{P}\left(\operatorname{deg}(v) \geq m_{v}, v \in[k]\right)=2^{-\sum_{v} m_{v}}\left(1+o\left(n^{-\alpha}\right)\right),
$$

as required.

\section{Proof of Proposition 2.1}

By Corollary 3.4 we can study vertex degrees in $T^{(n)}$ and derive conclusions about the variables $X_{i}^{(n)}, X_{\geq i}^{(n)}, i \in \mathbb{Z}$. Recall that we write $\operatorname{deg}(v)=\operatorname{deg}_{T^{(n)}}(v)$, for $v \in[n]$.

Lemma 5.1. For any $k \in \mathbb{N}$ and integers $m_{1}, \ldots, m_{k}$,

$$
\mathbf{P}\left(\operatorname{deg}(u)=m_{u}, u \in[k]\right)=\sum_{j=0}^{k} \sum_{\substack{S \subset[k] \\|S|=j}}(-1)^{j} \mathbf{P}\left(\operatorname{deg}(u) \geq m_{u}+\mathbf{1}_{[u \in S]}, u \in[k]\right) .
$$


Furthermore, for $k^{\prime} \in \mathbb{N}$ and integers $m_{k+1}, \ldots m_{k+k^{\prime}}$,

$$
\begin{aligned}
& \mathbf{P}\left(\operatorname{deg}(u)=m_{u}, \operatorname{deg}(v) \geq m_{v}, 1 \leq u \leq k<v \leq k+k^{\prime}\right) \\
= & \sum_{j=0}^{k} \sum_{\substack{S \subset[k] \\
|S|=j}}(-1)^{j} \mathbf{P}\left(\operatorname{deg}(v) \geq m_{v}+\mathbf{1}_{[v \in S]}, v \in\left[k+k^{\prime}\right]\right) .
\end{aligned}
$$

Proof. The second equation follows by intersecting the event $\left\{\operatorname{deg}(v) \geq m_{v}, k<v \leq k+k^{\prime}\right\}$ along all probabilities in the first equation. The first is straightforwardly proved using the inclusion-exclusion principle.

We are now ready to prove Proposition 2.1.

Proof of Proposition 2.1. Let $c \in(0,2)$ and $K \in \mathbb{N}$. Let $i<i^{\prime}$ be integers such that $0<i+\log _{n}<i^{\prime}+\log _{n}<c \ln n$ and let $a_{j}, i \leq j \leq i^{\prime}$ be non-negative integers with $a_{i}+\cdots a_{i^{\prime}}=K$. We are interested in the factorial moments $\mathbf{E}\left[\left(X_{\geq i^{\prime}}^{(n)}\right)_{a_{i^{\prime}}} \prod_{i \leq k<i^{\prime}}\left(X_{k}^{(n)}\right)_{a_{k}}\right]$.

For $i \leq k \leq i^{\prime}$, for each $v$ with $\sum_{l=i}^{k-1} a_{l}<v \leq \sum_{l=i}^{k} a_{l}$ let $m_{v}=\lfloor\log n\rfloor+k$. Let $K^{\prime}=K-a_{i^{\prime}}$, by Corollary 3.4 and the exchangeability of the vertex degrees of $T^{(n)}$,

$$
\begin{aligned}
\mathbf{E}\left[\left(X_{\geq i^{\prime}}^{(n)}\right)_{a_{i^{\prime}}} \prod_{i \leq k<i^{\prime}}\left(X_{k}^{(n)}\right)_{a_{k}}\right] & =(n)_{K} \mathbf{P}\left(\operatorname{deg}(u)=m_{u}, \operatorname{deg}(v) \geq m_{v}, 1 \leq u \leq K^{\prime}<v \leq K\right) \\
& =(n)_{K} \sum_{l=0}^{K^{\prime}} \sum_{\substack{S \subset\left[K^{\prime}\right] \\
|S|=l}}(-1)^{l} \mathbf{P}\left(\operatorname{deg}(v) \geq m_{v}+\mathbf{1}_{[v \in S]}, v \in[K]\right),
\end{aligned}
$$

the last equality by Lemma 5.1. At this point we can apply Proposition 4.2 to each of the terms. Since $m_{v} \leq c \ln n$ for $v \in[K]$, there is $\alpha^{\prime}=\alpha^{\prime}(c, K)>0$ such that

$$
\begin{aligned}
& \sum_{l=0}^{K^{\prime}} \sum_{\substack{S \subset\left[K^{\prime}\right] \\
|S|=l}}(-1)^{l} \mathbf{P}\left(\operatorname{deg}(v) \geq m_{v}+\mathbf{1}_{[v \in S]}, v \in[K]\right) \\
= & \sum_{l=0}^{K^{\prime}} \sum_{\substack{S \subset\left[K^{\prime}\right] \\
|S|=l}}(-1)^{l} 2^{-l-\sum_{v} m_{v}}\left(1+o\left(n^{-\alpha^{\prime}}\right)\right) \\
= & 2^{-\sum_{v} m_{v}}\left(1+o\left(n^{-\alpha^{\prime}}\right)\right) \sum_{l=0}^{K^{\prime}} \sum_{\substack{S \subset\left[K^{\prime}\right] \\
|S|=l}}(-1)^{l} 2^{-l} \\
= & 2^{-K^{\prime}-\sum_{v} m_{v}}\left(1+o\left(n^{-\alpha^{\prime}}\right)\right) .
\end{aligned}
$$

Using that $(n)_{K}=n^{K}\left(1+o\left(n^{-1}\right)\right)$, we get

$$
\mathbf{E}\left[\left(X_{\geq i^{\prime}}^{(n)}\right)_{a^{\prime}} \prod_{i \leq k<i^{\prime}}\left(X_{k}^{(n)}\right)_{a_{k}}\right]=2^{K \log n-K^{\prime}-\sum_{v=1}^{K} m_{v}}\left(1+o\left(n^{-\alpha}\right)\right) ;
$$


where $\alpha=\min \left\{\alpha^{\prime}, 1\right\}$. Finally, to complete the proof, note that

$$
\begin{aligned}
K \log n-K^{\prime}-\sum_{v=1}^{K} m_{v} & =\sum_{v=K^{\prime}+1}^{K}\left(\log n-m_{v}\right)+\sum_{v=1}^{K^{\prime}}\left(\log n-1-m_{v}\right) \\
& =\left(-i^{\prime}+\varepsilon_{n}\right) a_{i^{\prime}}+\sum_{k=i}^{i^{\prime}-1}\left(-k-1+\varepsilon_{n}\right) a_{k} .
\end{aligned}
$$

\section{Proofs of the MAIN TheOrems}

Proof of Theorem 1.2. By Theorem 11.1.VII of [4], weak convergence in $\mathcal{M}_{\mathbb{Z}^{*}}^{\#}$ is equivalent to convergence of FDD's, that is, convergence of every finite family of bounded continuity sets; see Definition 11.1.IV of [4]. For any point process $\xi$ on $\mathbb{Z}$ and any $i \in \mathbb{Z}$, we have that $\mathbb{Z} \cap[i, \infty)$ is a bounded stochastic continuity set for the underlying measure of $\xi$ in $\mathcal{M}_{\mathbb{Z}^{*}}^{\#}$. Thus, any FDD of $\xi$ can be recovered from suitable marginals of the joint distribution of $\left(\xi(i), \ldots, \xi\left(i-1^{\prime}\right), \xi[i, \infty)\right)$ for some $i<i^{\prime} \in \mathbb{Z}$.

Let $\varepsilon \in[0,1]$ and $\left(n_{l}\right)_{l \geq 1}$ be an increasing sequence with $\varepsilon_{n_{l}} \rightarrow \varepsilon$. The goal then is to prove that, for any integers $i<i^{\prime}$, the joint distribution of

$$
X_{i}^{\left(n_{l}\right)}, \ldots, X_{i^{\prime}-1}^{\left(n_{l}\right)}, X_{\geq i^{\prime}}^{\left(n_{l}\right)}
$$

converges to the joint distribution of

$$
\mathcal{P}^{\varepsilon}(i), \ldots, \mathcal{P}^{\varepsilon}\left(i^{\prime}-1\right), \mathcal{P}^{\varepsilon}\left[i^{\prime}, \infty\right),
$$

that is, to the law of independent Poisson r.v.'s with parameters $2^{-i-1+\varepsilon}, \ldots, 2^{-i^{\prime}-2+\varepsilon}, 2^{-i^{\prime}+\varepsilon}$.

We compute the limit of the factorial moments of $X_{i}^{\left(n_{l}\right)}, \ldots, X_{i^{\prime}-1}^{\left(n_{l}\right)}, X_{\geq i^{\prime}}^{\left(n_{l}\right)}$. For any nonnegative integers $a_{i}, \ldots, a_{i^{\prime}}$, by Proposition 2.1,

$$
\begin{aligned}
\mathbf{E}\left[\left(X_{\geq i^{\prime}}^{(n)} a_{i^{\prime}} \prod_{i \leq k<i^{\prime}}\left(X_{k}^{(n)}\right)_{a_{k}}\right]\right. & =\left(2^{-i^{\prime}+\varepsilon_{n}}\right)^{a_{i^{\prime}}} \prod_{i \leq k<i^{\prime}}\left(2^{-(k+1)+\varepsilon_{n}}\right)^{a_{k}}\left(1+o\left(n^{-\alpha}\right)\right) \\
& \rightarrow\left(2^{-i^{\prime}+\varepsilon}\right)^{a_{i^{\prime}}} \prod_{i \leq k<i^{\prime}}\left(2^{-(k+1)+\varepsilon}\right)^{a_{k}}
\end{aligned}
$$

as $n_{l} \rightarrow \infty$. The limit correspond to the factorial moment

$$
\mathbf{E}\left[\left(\mathcal{P}^{\varepsilon}\left[i^{\prime}, \infty\right)\right)_{a_{i}^{\prime}} \prod_{i \leq k<i^{\prime}}\left(\mathcal{P}^{\varepsilon}(k)\right)_{a_{k}}\right] .
$$

The result follows (by, e.g. Theorem 6.10 of [9]).

Proof of Theorem 1.3. Since $\left\{\Delta_{n} \geq\lfloor\log n\rfloor+i\right\}=\left\{X_{\geq i}^{(n)}>0\right\}$, we need only to estimate $\mathbf{P}\left(X_{\geq i}^{(n)}>0\right)$. If $i=O(1)$, then $\exp \left\{-2^{-i+\varepsilon_{n}}\right\}=O(1)$ and so it suffices to prove that

$$
\left.\mathbf{P}\left(X_{\geq i}^{(n)}=0\right)-\exp \left\{-2^{-i+\varepsilon_{n}}\right\}\right) \rightarrow 0,
$$

as $n \rightarrow \infty$. This follows from Theorem 1.2 and the subsubsequence principle. Suppose that there exists $\delta>0$ and a subsequence $n_{k}$ for which $\left|\mathbf{P}\left(X_{\geq i}^{\left(n_{k}\right)}=0\right)-\exp \left\{-2^{-i+\varepsilon_{n_{k}}}\right\}\right|>\delta$. Since $\left\{\varepsilon_{n_{k}}\right\}_{k \geq 1}$ is a bounded set there is a subsubsequence $n_{k_{l}}$ such that $\varepsilon_{n_{k_{l}}} \rightarrow \varepsilon$ for some $\varepsilon \in[0,1]$. By Theorem 1.2, $\mathbf{P}\left(X_{\geq i}^{\left(n_{k_{l}}\right)}=0\right) \rightarrow \exp \left\{-2^{-i+\varepsilon}\right\}$; this contradicts our assumption on the subsequence $n_{k}$. 
Now consider the case $i \rightarrow \infty$ with $i+\log _{n}<2 \ln n$. By a standard inclusion-exclusion argument (see, e.g., [3] Corollary 1.11),

$$
\mathbf{P}\left(X_{\geq i}^{(n)}=0\right)=\sum_{r=0}^{n}(-1)^{r} \frac{\mathbf{E}\left[\left(X_{\geq i}^{(n)}\right)_{r}\right]}{r !},
$$

and this sum has the so called alternating inequalities property; this means that partial sums alternatively serve as upper and lower bounds for $\mathbf{P}\left(X_{\geq i}^{(n)}=0\right)$. Consequently ${ }^{1}$,

$$
\mathbf{E}\left[X_{\geq i}^{(n)}\right]-\frac{1}{2} \mathbf{E}\left[\left(X_{\geq i}^{(n)}\right)_{2}\right] \leq \mathbf{P}\left(X_{\geq i}^{(n)}>0\right) \leq \mathbf{E}\left[X_{\geq i}^{(n)}\right] .
$$

Using Proposition 2.1 and the fact that $i \rightarrow \infty$, we have that $\mathbf{E}\left[X_{\geq i}^{(n)}\right]=2^{-i+\varepsilon_{n}}(1+o(1))$ and

$$
\mathbf{E}\left[X_{\geq i}^{(n)}\right]-\frac{1}{2} \mathbf{E}\left[\left(X_{\geq i}^{(n)}\right)_{2}\right]=2^{-i+\varepsilon_{n}}(1+o(1))=\left(1-\exp \left\{-2^{-i+\varepsilon_{n}}\right\}\right)(1+o(1)) .
$$

The result follows.

Proof of Theorem 1.4. We again use the method of moments. By Theorem 1.24 of [3], it suffices to prove that, as $n \rightarrow \infty$

$$
\mathbf{E}\left[\left(X_{i}^{(n)}\right)_{a}\right]-\left(2^{-i-1+\varepsilon_{n}}\right)^{a}=o\left(2^{-\left(i+1-\varepsilon_{n}\right) b}\right),
$$

for all fixed $1 \leq a \leq b$. Since $i=o(\ln n)$, we have that $2^{-i-1+\varepsilon_{n}}=n^{o(1)}$. On the other hand, by Proposition 2.1 there is $\alpha>0$ such that

$$
\mathbf{E}\left[\left(X_{i}^{(n)}\right)_{a}\right]-\left(2^{-i-1+\varepsilon_{n}}\right)^{a}=o\left(n^{-\alpha} 2^{-\left(i+\varepsilon_{n}\right) a}\right)=n^{-\alpha+o(1)}=o\left(n^{o(1)}\right) .
$$

Therefore, condition (9) is satisfied and the proof is complete.

Acknowledgements. Laura Eslava would like to thank Henning Sulzbach for some very helpful discussions.

\section{REFERENCES}

[1] Louigi Addario-Berry. Partition functions of discrete coalescents: from Cayley's formula to Frieze's $\zeta(3)$ limit theorem. In XI Symposium on Probability and Stochastic Processes, volume 68 of Progress in Probability, Basel, 2015. Birkhauser.

[2] Nathanaël Berestycki. Recent progress in coalescent theory, volume 16 of Ensaios Matemáticos [Mathematical Surveys]. Sociedade Brasileira de Matemática, Rio de Janeiro, 2009. ISBN 978-85-85818-40-1.

[3] Béla Bollobás. Random graphs, volume 73 of Cambridge Studies in Advanced Mathematics. Cambridge University Press, Cambridge, second edition, 2001. ISBN 0-52180920-7; 0-521-79722-5. doi: 10.1017/CBO9780511814068. URL http://dx.doi.org/ 10.1017/СB09780511814068.

[4] D. J. Daley and D. Vere-Jones. An introduction to the theory of point processes. Vol. II. Probability and its Applications (New York). Springer, New York, second edition, 2008. ISBN 978-0-387-21337-8. doi: 10.1007/978-0-387-49835-5. URL http://dx . doi.org/10.1007/978-0-387-49835-5. General theory and structure.

\footnotetext{
${ }^{1}$ A similar lower bound for $\mathbf{P}\left(X_{\geq i}^{(n)}>0\right)$ could be obtained from Paley-Zigmund's inequality.
} 
[5] L. Devroye. Branching processes in the analysis of the heights of trees. Acta Inform., 24(3):277-298, 1987. ISSN 0001-5903. doi: 10.1007/BF00265991. URL http://dx . doi.org/10.1007/BF00265991.

[6] Luc Devroye and Jiang Lu. The strong convergence of maximal degrees in uniform random recursive trees and dags. Random Structures Algorithms, 7(1):1-14, 1995. ISSN 1042-9832. doi: 10.1002/rsa.3240070102. URL http://dx.doi.org/10.1002/ rsa. 3240070102.

[7] William Goh and Eric Schmutz. Limit distribution for the maximum degree of a random recursive tree. J. Comput. Appl. Math., 142(1):61-82, 2002. ISSN 03770427. doi: 10.1016/S0377-0427(01)00460-5. URL http://dx.doi.org/10.1016/ S0377-0427(01)00460-5. Probabilistic methods in combinatorics and combinatorial optimization.

[8] Svante Janson. Asymptotic degree distribution in random recursive trees. Random Structures Algorithms, 26(1-2):69-83, 2005. ISSN 1042-9832. doi: 10.1002/rsa.20046. URL http://dx.doi.org/10.1002/rsa.20046.

[9] Svante Janson, Tomasz Łuczak, and Andrzej Rucinski. Random graphs. WileyInterscience Series in Discrete Mathematics and Optimization. Wiley-Interscience, New York, 2000. ISBN 0-471-17541-2. doi: 10.1002/9781118032718. URL http: //dx.doi.org/10.1002/9781118032718.

[10] Kumar Joag-Dev and Frank Proschan. Negative association of random variables, with applications. Ann. Statist., 11(1):286-295, 1983. ISSN 0090-5364. doi: 10.1214/aos/ 1176346079. URL http://dx.doi.org/10.1214/aos/1176346079.

[11] Hosam M. Mahmoud and R. T. Smythe. Asymptotic joint normality of outdegrees of nodes in random recursive trees. Random Structures Algorithms, 3(3):255-266, 1992. ISSN 1042-9832. doi: 10.1002/rsa.3240030305. URL http://dx.doi.org/10.1002/ rsa. 3240030305.

[12] A. Meir and J. W. Moon. Recursive trees with no nodes of out-degree one. Congr. Numerantium 66: 49-62,1988.

[13] Hwa Sung Na and Anatol Rapoport. Distribution of nodes of a tree by degree. Math. Biosci., 6:313-329, 1970. ISSN 0025-5564.

[14] Boris Pittel. Note on the heights of random recursive trees and random $m$-ary search trees. Random Structures Algorithms, 5(2):337-347, 1994. ISSN 1042-9832. doi: 10. 1002/rsa.3240050207. URL http://dx.doi.org/10.1002/rsa.3240050207.

[15] Jerzy Szymański. On the maximum degree and the height of a random recursive tree. In Random graphs '87 (Poznań, 1987), pages 313-324. Wiley, Chichester, 1990.

E-mail address: louigi@problab.ca

E-mail address: laura.eslavafernandez@mail.mcgill.ca

Department of Mathematics and Statistics, McGill University, Montreal, Canada 